\title{
Novel Recursive Approximation for Fractional Nonlinear Equations within Caputo-Fabrizio Operator
}

\author{
Mehmet Yavuz ${ }^{1, *}$ \\ ${ }^{1}$ Department of Mathematics-Computer Sciences, Necmettin Erbakan University, Konya, Turkey
}

\begin{abstract}
This study displays a novel method for solving time-fractional nonlinear partial differential equations. The suggested method namely Laplace homotopy method (LHM) is considered with Caputo-Fabrizio fractional derivative operator. In order to show the efficiency and accuracy of the mentioned method, we have applied it to time-fractional nonlinear Klein-Gordon equation. Comparisons between obtained solutions and the exact solutions have been made and the analysis shows that recommended solution method presents a rapid convergence to the exact solutions of the problems.
\end{abstract}

\section{Introduction}

In the last decades, fractional calculus were applied to various fields of mathematical and physical analysis such as modelling some common viruses, estimation financial processes, diffusion, relaxation processes and so on [1-11]. Especially, modelling the nonlinear problems in these areas with fractional differential equations was extensively used. In order to find out the efficiency and superiority of the fractional differential equations, a lot of studies were emerged by some scientists [12-17]. On the other hand, some important fractional derivative operators have been developed such as Caputo-Fabrizio [18] and Atangana-Baleanu [19]. These operators are very important to model the complex nonlinear fractional dynamical systems and to solve them. In recent years, some scientists have preferred these operators to other fractional operators which have a singularity [11, 20-23]. In this study, we also use the Caputo-Fabrizio fractional operator to solve nonlinear KleinGordon equation by considering the Laplace homotopy method.

\section{Caputo-Fabrizio Operator and its Main Properties}

Definition 1. The Caputo-Fabrizio fractional derivative in Caputo sense (CFC) is defined by [18]

\footnotetext{
*Corresponding author: mehmetyavuz@konya.edu.tr
} 


$$
{ }_{0}^{C F C} D_{t}^{\beta} f(t)=\frac{M(\beta)}{1-\beta} \int_{0}^{t} f^{\prime}(\tau) \exp \left[-\frac{\beta(t-\tau)}{1-\beta}\right] d \tau, \quad 0<\beta \leq 1,
$$

where $M(\beta)$ is a normalization function such that $M(0)=M(1)=1$. The CF definition can be applied for the functions which do not belong to $H^{1}(a, b)$ and the kernel does not have singularity for $t=\tau$ unlike the Caputo fractional derivative.

Definition 2. The Laplace transform (LT) of ${ }_{0}^{C F C} D_{t}^{\beta} f(t)$ is given by $[18,24]$ :

$$
\begin{aligned}
\mathcal{L}\left\{{ }_{0}{ }_{0}^{C F C} D_{t}^{(\beta+n)} f(t)\right\}(s) & =\frac{1}{1-\beta} \mathcal{L}\left\{f^{(\beta+n)}(t)\right\} \mathcal{L}\left\{\exp \left[-\frac{\beta t}{1-\beta}\right]\right\} \\
& =\frac{s^{n+1} L\{f(t)\}-s^{n} f(0)-s^{n-1} f^{\prime}(0)-\cdots-f^{(n)}(0)}{s+\beta(1-s)} .
\end{aligned}
$$

Definition 3. Let $0<\beta<1$. The CFC fractional integral of order $\beta$ is defined by $[25,26]$ :

$$
{ }_{0}^{C F C} I^{\beta} f(t)=\frac{2(1-\beta)}{(2-\beta) M(\beta)} f(t)+\frac{2 \beta}{(2-\beta) M(\beta)} \int_{0}^{t} f(\tau) d \tau, \quad t \geq 0 .
$$

\section{Role of the Suggested Fractional Operator in the Fundamental Method}

Consider the following nonlinear FPDE:

$$
{ }^{C F C} D_{t}^{\beta} \phi(x, t)+\mu(\phi(x, t))+\omega(\phi(x, t))=v(x, t), \quad(x, t) \in[0,1] \times[0, T], \quad 0<\beta \leq 1,
$$

with initial condition

$$
\phi(x, 0)=k(x)
$$

where ${ }^{C F C} D_{t}^{\beta}$ shows the Caputo-Fabrizio-Caputo fractional derivative of order $\beta$, $\omega(\phi(x, t))$ is a non-linear operator and $v(x, t)$ is a known function and $\phi(x, t)$ is an unknown function. Using the LT of the CF operator we define $\mathcal{L}\{\phi(x, t)\}(s)=\Delta(x, s)$. Then we have the following homotopies:

$$
\Delta(x, s)=\left(\frac{\beta+s(1-\beta)}{s}\right)[\tilde{v}(x, s)-\mathcal{L}\{\mu(\phi(x, t))+\omega(\phi(x, t))\}]+\frac{1}{s}\left(\phi_{0}(x)\right) .
$$

where $\Delta(x, s)=\mathcal{L}\{\phi(x, t)\}$ and $\tilde{v}(x, s)=\mathcal{L}\{v(x, t)\}$. Applying the suggested method we obtain the approximate solution as

$$
\Delta(x, s)=\sum_{m=0}^{\infty} p^{m} \Delta_{m}(x, s), m=0,1,2, \ldots
$$

where $\Delta_{m}(x, s)$ values are known functions. The nonlinear term $\omega(\phi(x, t))$ in Eq. (4) can be evaluate as

$$
\omega(\phi(x, t))=\sum_{m=0}^{\infty} p^{m} \eta_{m}(x, t)
$$

and the polynomials $\eta_{m}(x, t)$ are given in [27] as 


$$
\eta_{m}\left(\phi_{0}, \phi_{1}, \ldots, \phi_{m}\right)=\frac{1}{m !} \frac{\partial^{m}}{\partial p^{m}}\left[\omega\left(\sum_{i=0}^{\infty} p^{i} \phi_{i}\right)\right]_{p=0}, m=0,1,2, \ldots .
$$

Substituting Eqs.(8) and (9) into Eq.(6) we construct the solution series for the main problem.

$$
\begin{aligned}
\sum_{m=0}^{\infty} p^{m} \Delta_{m}(x, s) & =-p\left[\left(\frac{\beta+s(1-\beta)}{s}\right)\left(\mathcal{L}\left\{\sum_{m=0}^{\infty} p^{m} \Delta_{m}(x, t)+\sum_{m=0}^{\infty} p^{m} \eta_{m}(x, t)\right\}\right)\right] \\
& +\frac{1}{s}\left(\phi_{0}(x)\right)+\left(\frac{\beta+s(1-\beta)}{s}\right) \tilde{v}(x, s) .
\end{aligned}
$$

By equating the same powers of $p$, we construct the homotopy steps of as

$$
\begin{aligned}
& p^{0}: \Delta_{0}(x, s)=\frac{1}{s}\left(\phi_{0}(x)\right)+\left(\frac{\beta+s(1-\beta)}{s}\right) \tilde{v}(x, s), \\
& p^{1}: \Delta_{1}(x, s)=-\left(\frac{\beta+s(1-\beta)}{s}\right) \mathcal{L}\left\{\mu\left(\phi_{0}(x, t)\right)+\omega\left(\phi_{0}(x, t)\right)\right\}, \\
& p^{2}: \Delta_{2}(x, s)=-\left(\frac{\beta+s(1-\beta)}{s}\right) \mathcal{L}\left\{\mu\left(\phi_{1}(x, t)\right)+\omega\left(\phi_{1}(x, t)\right)\right\}, \\
& \vdots \\
& p^{n+1}: \Delta_{n+1}(x, s)=-\left(\frac{\beta+s(1-\beta)}{s}\right) \mathcal{L}\left\{\mu\left(\phi_{n}(x, t)\right)+\omega\left(\phi_{n}(x, t)\right)\right\} .
\end{aligned}
$$

Considering $p \rightarrow 1$, we can see Eq.(11) gives the approximate solution for problem (10) and the solution is given by

$$
\mathfrak{I}_{n}(x, s)=\sum_{j=0}^{n} \Delta_{j}(x, s) .
$$

Taking the inverse Laplace transform of the sum (12), we have the approximate solution

$$
\phi_{\text {approx }}(x, t) \cong \phi_{n}(x, t)=\mathcal{L}^{-1}\left\{\mathfrak{J}_{n}(x, s)\right\} .
$$

\section{Solution of Nonlinear Fractional Klein-Gordon Equation}

Consider the fractional nonlinear homogeneous Klein-Gordon equation (KGE) [28]

$$
{ }^{C F C} D_{t}^{\beta} \phi(x, t)-\phi_{x x}(x, t)+\phi^{2}(x, t)=0, \quad x \in R, \quad t>0, \quad 0<\beta \leq 1,
$$

subject to the initial condition

$$
\phi(x, 0)=1+\sin x, \quad x \in R .
$$

Firstly, applying the mentioned method in Section 3, to Eq.(13) with the initial condition (14), we obtain the approximate solution as

$$
\Delta(x, s)=\left(\frac{\beta+s(1-\beta)}{s}\right) \mathcal{L}\left\{\phi_{x x}(x, t)-\phi^{2}(x, t)\right\}+\frac{1}{s}[\phi(x, 0)] .
$$

Then, we consider the inverse Laplace transform of Eq.(15), we have

$$
\phi(x, t)=\phi(x, 0)+\mathcal{L}^{-1}\left\{\left(\frac{\beta+s(1-\beta)}{s}\right) \mathcal{L}\left\{\phi_{x x}(x, t)-\phi^{2}(x, t)\right\}\right\} .
$$


The nonlinear polynomials $\eta_{m}(x, t)$ are given in [27] as

$$
\begin{aligned}
\eta_{0}(\phi) & =(1+\sin x)^{2} \\
\eta_{1}(\phi) & =-2(1+\sin x)\left(\sin ^{2} x+3 \sin x+1\right)(\beta t-\beta+1), \\
\eta_{2}(\phi) & =\left(\left(\sin ^{2} x+3 \sin x+1\right)(\beta t-\beta+1)\right)^{2}+2(1+\sin x)(3 \sin x-2 \cos 2 x)(\beta t-\beta+1) \\
& +4(1+\sin x)^{2}\left(\sin ^{2} x+3 \sin x+1\right)\left(\beta^{2}-2 \beta+1+\frac{\beta^{2} t^{2}}{2}-2\left(\beta^{2}-\beta\right) t\right),
\end{aligned}
$$

Moreover, we use the series solution in Eq.(10), we can write

$$
\sum_{m=0}^{\infty} p^{m} \Delta_{m}(x, s)=-p\left[\left(\frac{\beta+s(1-\beta)}{s}\right) \mathcal{L}\left\{\sum_{m=0}^{\infty} p^{m} \Delta_{m}(x, t)+\sum_{m=0}^{\infty} p^{m} \eta_{m}(x, t)\right\}\right]+\frac{1}{s}\left(\phi_{0}(x)\right) .
$$

By comparing the coefficients of powers of $p$, we construct the homotopy steps as in Eq.(11) and taking the inverse LT of obtained values, we have the iteration steps as

$$
\begin{aligned}
& \phi_{0}(x, t)=1+\sin x, \\
& \begin{aligned}
\phi_{1}(x, t)= & -\left(\sin ^{2} x+3 \sin x+1\right)(\beta t-\beta+1), \\
\phi_{2}(x, t)= & 2(1+\sin x)\left(\sin ^{2} x+3 \sin x+1\right)\left(\beta^{2}-2 \beta+1+\frac{\beta^{2} t^{2}}{2}-2\left(\beta^{2}-\beta\right) t\right) \\
& +(3 \sin x-2 \cos 2 x)(\beta t-\beta+1), \\
\vdots & \phi_{n+1}(x, t)=\mathcal{L}^{-1}\left\{\left(\frac{\beta+s(1-\beta)}{s}\right) \mathcal{L}\left\{\mu\left(\phi_{n}(x, t)\right)+\omega\left(\phi_{n}(x, t)\right)\right\}\right\} .
\end{aligned}
\end{aligned}
$$

Finally, considering the sum (19), we have the approximate solution

$$
\begin{aligned}
\phi_{\text {approx }}(x, t) \cong & \phi_{n}(x, t)=1+\sin x-\left(\sin ^{2} x+2 \cos 2 x+1\right)(\beta t-\beta+1) \\
& +2(1+\sin x)\left(\sin ^{2} x+3 \sin x+1\right)\left(\beta^{2}-2 \beta+1+\frac{\beta^{2} t^{2}}{2}-2\left(\beta^{2}-\beta\right) t\right)+\ldots .
\end{aligned}
$$

Following figures show the solution functions for some different values of $x, t$ and fractional parameter $\beta$.
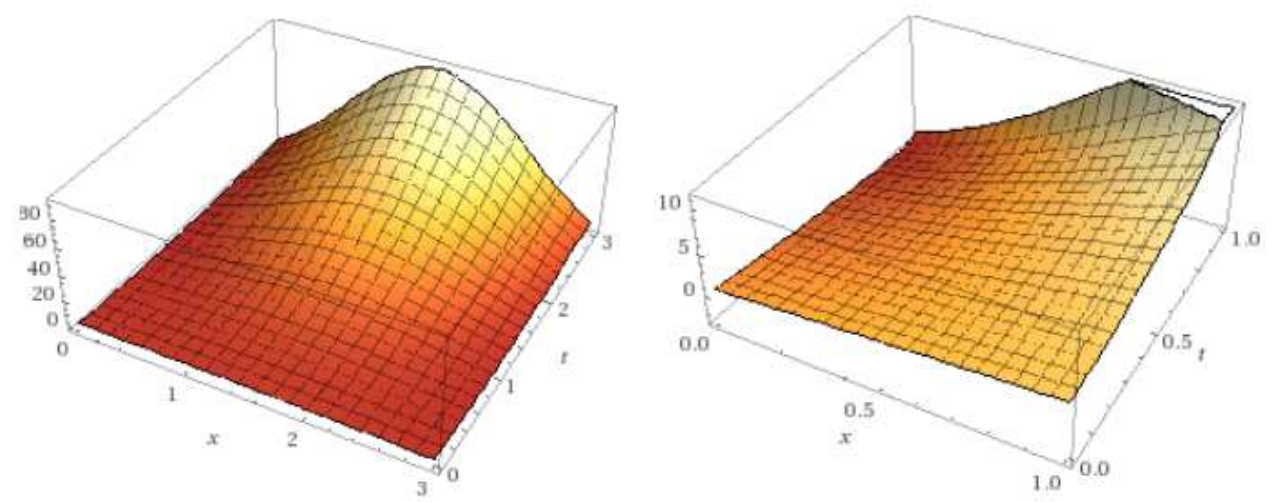

Fig.1. Solutions of Eq.(13) for $\beta=1$ (left) $\beta=0.85$ (right) 

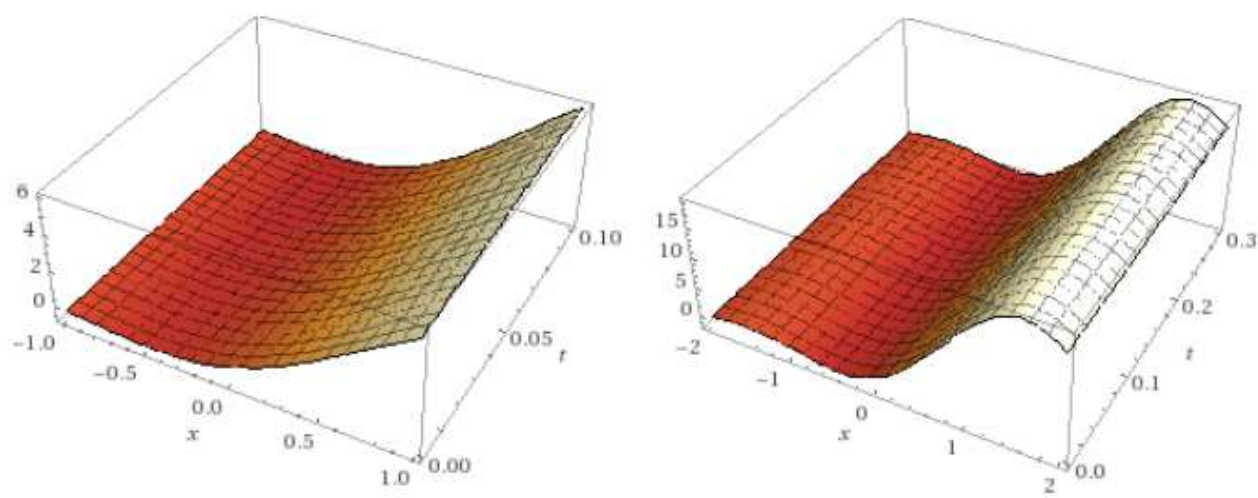

Fig.2. Solutions of Eq.(13) for $\beta=0.5$ (left) $\beta=0.1$ (right)
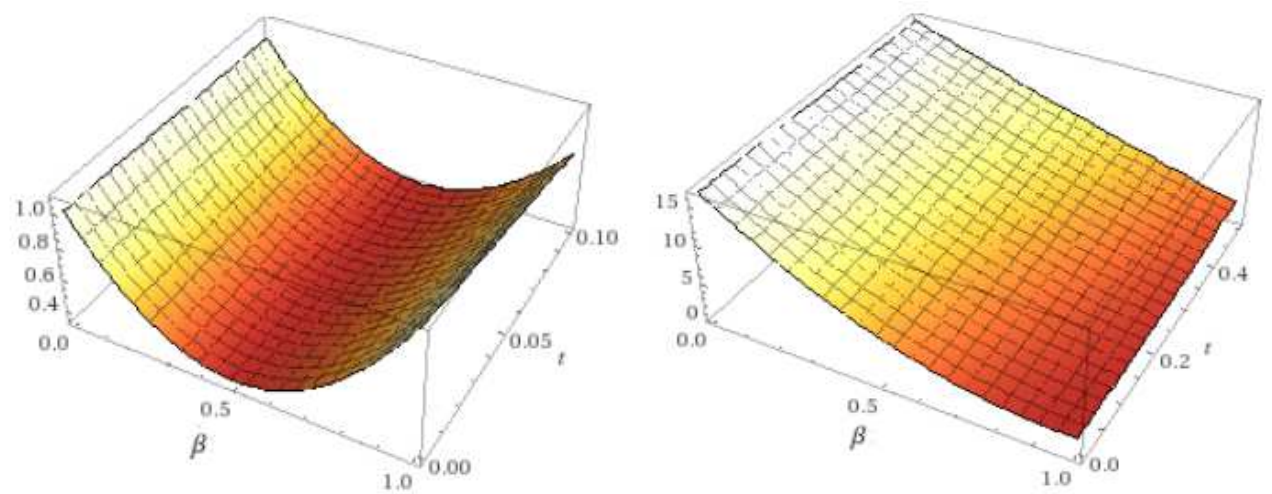

Fig.3. Solutions of Eq.(13) for $x=0.1$ (left) $x=1$ (right)

\section{Conclusions}

In this study, Laplace homotopy method which is defined with a new fractional operator is considered. This method presents an efficient solution for nonlinear FDEs without discretization. Therefore, this method can be applied to nonlinear FDEs easily. The nonlinear terms in the equation are evaluated by using Adomian polynomials. In this context, we obtain the approximate-analytical solution of nonlinear KGE of fractional order by using the LHM. The results obtained in this study carried out that the method is very efficient and accurate method for solving the fractional nonlinear homogeneous KGE. Maple software has been used for obtaining the numerical computations and presenting the solution plots with respect to various values of the variables.

\section{References}

1. T. Abdeljawad, D. Baleanu, Integration by parts and its applications of a new nonlocal fractional derivative with Mittag-Leffler nonsingular kernel. Journal of Nonlinear Sciences and Applications, 10(2017), pp. 1098-1107, (2017). 
2. D. Avci, B.B. Iskender Eroglu, N. Ozdemir, Conformable heat equation on a radial symmetric plate. Thermal Science, 21(2), pp. 819-826, (2017).

3. E. Bonyah, A. Atangana, M.A. Khan, Modeling the spread of computer virus via Caputo fractional derivative and the beta-derivative. Asia Pacific Journal on Computational Engineering, 4(1), p. 1, (2017).

4. Y. Çenesiz, D. Baleanu, A. Kurt, O. Tasbozan, New exact solutions of Burgers' type equations with conformable derivative. Waves in Random and Complex Media, 27(1), pp. 103-116, (2017).

5. F. Evirgen, Analyze the optimal solutions of optimization problems by means of fractional gradient based system using VIM. An International Journal of Optimization and Control, 6(2), pp. 75-83, (2016).

6. J.F. Gomez-Aguilar, H. Yépez-Martínez, J. Torres-Jiménez, T. Córdova-Fraga, R.F. Escobar-Jiménez, V.H. Olivares-Peregrino, Homotopy perturbation transform method for nonlinear differential equations involving to fractional operator with exponential kernel. Advances in Difference Equations, 2017(1), p. 68, (2017).

7. J. Hristov, Steady-state heat conduction in a medium with spatial non-singular fading memory: derivation of Caputo-Fabrizio space-fractional derivative with Jeffrey's kernel and analytical solutions. Thermal science, 21(2), pp. 827-839, (2017).

8. M. Yavuz, Novel solution methods for initial boundary value problems of fractional order with conformable differentiation. An International Journal of Optimization and Control: Theories \& Applications (IJOCTA), 8(1), pp. 1-7, (2018).

9. M. Yavuz, N. Ozdemir, Numerical inverse Laplace homotopy technique for fractional heat equations. Thermal Science, 22(2), pp. 185-194, (2018).

10. M. Yavuz, N. Ozdemir A different approach to the European option pricing model with new fractional operator. Mathematical Modelling of Natural Phenomena, 13(1), pp. 1-12, (2018).

11. M. Yavuz, N. Ozdemir, H.M. Baskonus, Solutions of partial differential equations using the fractional operator involving Mittag-Leffler kernel. The European Physical Journal Plus, 133(6), p. 215, (2018).

12. N. Özdemir, M. Yavuz, Numerical Solution of Fractional Black-Scholes Equation by Using the Multivariate Padé Approximation. Acta Physica Polonica A, 132(3), pp. 1050-1053, (2017).

13. H. Bulut, T.A. Sulaiman, H.M. Baskonus, Dark, bright optical and other solitons with conformable space-time fractional second-order spatiotemporal dispersion. Optik, 163, pp. 1-7, (2018).

14. H.M, Baskonus, T. Mekkaoui, Z. Hammouch, H. Bulut, Active control of a chaotic fractional order economic system. Entropy, 17(8), pp. 5771-5783, (2015).

15. A. Esen, Y. Ucar, N. Yagmurlu, O. Tasbozan, A Galerkin finite element method to solve fractional diffusion and fractional diffusion-wave equations. Mathematical Modelling and Analysis, 18(2), pp. 260-273, (2013).

16. A. Yokus, H.M. Baskonus, T.A. Sulaiman, H. Bulut, Numerical simulation and solutions of the two-component second order KdV evolutionarysystem. Numerical Methods for Partial Differential Equations, 34(1), pp. 211-227, (2018).

17. D. Kaya, S. Gülbahar, A. Yokuş, M. Gülbahar, Solutions of the fractional combined KdV$m K d V$ equation with collocation method using radial basis function and their geometrical obstructions. Advances in Difference Equations, 2018(1), p. 77, (2018).

18. M. Caputo, M. Fabrizio, A new definition of fractional derivative without singular kernel. Progress in Fractional Differentiation and Applications, 1(2), pp. 1-13, (2015).

19. A. Atangana, D. Baleanu, New fractional derivatives with nonlocal and non-singular kernel: Theory and application to heat transfer model. Thermal Science, 20(2), pp. 763769, (2016).

20. N.A. Sheikh, F. Ali, M. Saqib, I. Khan, S.A.A. Jan, A comparative study of AtanganaBaleanu and Caputo-Fabrizio fractional derivatives to the convective flow of a generalized Casson fluid. The European Physical Journal Plus, 132(1), p. 54, (2017).

21. J.F. Gómez-Aguilar, R.F. Escobar-Jiménez, M.G. López-López, V.M. Alvarado-Martínez, Atangana-Baleanu fractional derivative applied to electromagnetic waves in dielectric media. Journal of Electromagnetic Waves and Applications, 30(15), pp. 1937-1952, (2016). 


\section{CMES-2018}

22. A. Atangana, D. Baleanu, Caputo-Fabrizio derivative applied to groundwater flow within confined aquifer. Journal of Engineering Mechanics, 143(5), p. D4016005, (2017).

23. M. Yavuz, N. Özdemir, European vanilla option pricing model of fractional order without singular kernel. Fractal and Fractional, 2(1), p. 3, (2018).

24. A. Atangana, B.S.T. Alkahtani, New model of groundwater flowing within a confine aquifer: application of Caputo-Fabrizio derivative. Arabian Journal of Geosciences, 9(1), p. $8,(2016)$.

25. J. Losada, J.J. Nieto, Properties of a new fractional derivative without singular kernel. Progress in Fractional Differentiation and Applications, 1(2), pp. 87-92, (2015).

26. D. Baleanu, B. Agheli, M.M. Al Qurashi, Fractional advection differential equation within Caputo and Caputo-Fabrizio derivatives. Advances in Mechanical Engineering, 8(12), p. 1687814016683305, (2016).

27. A. Ghorbani, Beyond Adomian polynomials: he polynomials. Chaos, Solitons \& Fractals, 39(3), pp. 1486-1492, (2009).

28. A.K. Golmankhaneh, A.K. Golmankhaneh, D. Baleanu, On nonlinear fractional KleinGordon equation. Signal Processing, 91(2011), pp. 446-451, (2011). 